\title{
Patient Motivation and Adherence to Postsurgery Rehabilitation Exercise Recommendations: The Influence of Physiotherapists' Autonomy-Supportive Behaviors
}

\author{
Derwin K. Chan, BSSc, Chris Lonsdale, PhD, Po Y. Ho, MD, Patrick S. Yung, MD, Kai M. Chan, MD
}

ABSTRACT. Chan DK, Lonsdale C, Ho PY, Yung PS, Chan KM. Patient motivation and adherence to postsurgery rehabilitation exercise recommendations: the influence of physiotherapists' autonomy-supportive behaviors. Arch Phys Med Rehabil 2009;90:1977-82.

Objective: To investigate the impact of physiotherapists' autonomy-supportive behaviors on patients' motivation and rehabilitation adherence after anterior cruciate ligament (ACL) reconstruction surgery.

Design: Retrospective study.

Setting: Outpatient orthopedic clinic of a university medical center.

Participants: Postsurgery ACL reconstruction patients $(\mathrm{N}=115$; minimum postsurgery interval, $6 \mathrm{mo}$; mean $\pm \mathrm{SD}$ postsurgery interval, $1.77 \pm 0.8 \mathrm{y}$ ).

Interventions: Not applicable.

Main Outcome Measures: Questionnaires measuring autonomy support from physiotherapists (Health Care Climate Questionnaire), treatment motivation (Treatment Self-Regulation Questionnaire), and rehabilitation adherence (adapted from the Sport Injury Rehabilitation Adherence Scale and the Patient Self-Report Scales of Their Home-Based Rehabilitation Adherence).

Results: Structural equation modeling analyses revealed that patients' treatment motivation mediated the relationship between physiotherapists' autonomy-supportive behaviors and rehabilitation adherence. Autonomy-supportive behavior positively predicted autonomous treatment motivation $(\beta=.22$, $P<.05)$. Rehabilitation adherence $\left(R^{2}=.28\right)$ was predicted positively by autonomous motivation $(\beta=.64, P<.05)$ and negatively predicted by controlled motivation $(\beta=-.28, P<.05)$.

Conclusions: These preliminary findings are promising and provide an empirical basis for further research to test the efficacy of autonomy support training designed to increase patients' rehabilitation adherence.

Key Words: Anterior cruciate ligament; Motivation; Rehabilitation.

(C) 2009 by the American Congress of Rehabilitation Medicine

From the Department of Orthopaedics and Traumatology, Faculty of Medicine, The Chinese University of Hong Kong, Shatin, Hong Kong, China (Chan DK, Ho, Yung, Chan KM); The Hong Kong Jockey Club Sports Medicine and Health Sciences Centre, Shatin, Hong Kong, China (Chan DK, Ho, Yung, Chan KM); University College Dublin, Dublin, Ireland (Lonsdale).

No commercial party having a direct financial interest in the results of the research supporting this article has or will confer a benefit on the authors or on any organization with which the authors are associated.

Correspondence to Chris Lonsdale, PhD, School of Public Health, Physiotherapy and Population Science, Health Sciences Bldg, University College Dublin, Belfield, Dublin 4, Ireland, e-mail: chris.lonsdale@ucd.ie. Reprints are not available from the author.

0003-9993/09/9012-00148\$36.00/0

doi:10.1016/j.apmr.2009.05.024
NTERIOR CRUCIATE LIGAMENT ruptures are among the most common severe sport injuries that require a long period of rehabilitation. ${ }^{1}$ To fully recover, patients need to undergo ACL reconstruction surgery, which is typically followed by a 6- to 12 -month rehabilitation period. ${ }^{2,3}$ Patients' compliance during this long period of rehabilitation may be problematic, ${ }^{4,5}$ with motivation representing one of the critical factors that impacts their adherence to prescribed treatment protocols. $^{6-12}$ In this study, we used SDT $^{13}$ as a framework from which to investigate the impact of physiotherapists' behavior on patients' treatment motivation and rehabilitation adherence after surgery to repair a ruptured ACL.

Understanding the dynamics of treatment motivation is an important goal for those who seek to enhance patients' adherence $^{11}$ and subsequent recovery from musculoskeletal injuries. ${ }^{14,15}$ Motivation of sports-injured patients ${ }^{16-20}$ to undertake rehabilitation has been found to be positively associated with attendance at rehabilitation sessions, completion of prescribed treatment protocols, self-rated adherence, and self-reported home exercise completion. However, Ryan et $\mathrm{al}^{11}$ argued that all forms of motivation to undergo rehabilitation do not necessarily facilitate treatment persistence. Indeed, they argued that the sources of motivation mattered, and introduced SDT to explain the motivational dynamics surrounding patient adherence to recommended treatment.

SDT $^{13,21}$ is a contemporary psychologic theory which proposes that one's behavior can be motivated by internal and external reasons. These reasons, also known as behavioral regulations, differ in terms of the level of autonomy experienced by the person and can be ordered on a continuum of self-determination (fig 1). At the most autonomous pole of the continuum, behaviors are regulated by intrinsic motivation where actions are performed because of interest, enjoyment, or both, regardless of the instrumental value of the behaviors. In line with traditional theories, ${ }^{13}$ SDT also describes behaviors that are underpinned by extrinsic motivation, whereby persons engage in activities in order to obtain a separable outcome, rather than for the inherent enjoyment or pleasure of the activities. In contrast to many theories, ${ }^{13}$ SDT proposes that extrinsic motivation can be divided into 4 categories, 2 of which are underpinned by controlled motivation (external and introjected

List of Abbreviations

$\begin{array}{ll}\text { ACL } & \text { anterior cruciate ligament } \\ \text { CFI } & \text { comparative fit index } \\ \text { HCCO } & \text { Health Care Climate Questionnaire } \\ \text { RMSEA } & \text { root mean squared error of approximation } \\ \text { SDT } & \text { self-determination theory } \\ \text { SEM } & \text { structural equation modeling } \\ \text { SRMR } & \text { standardized root mean square residual } \\ \text { TLI } & \text { Tucker-Lewis index } \\ \text { TSRO } & \text { Treatment Self-Regulation Questionnaire }\end{array}$




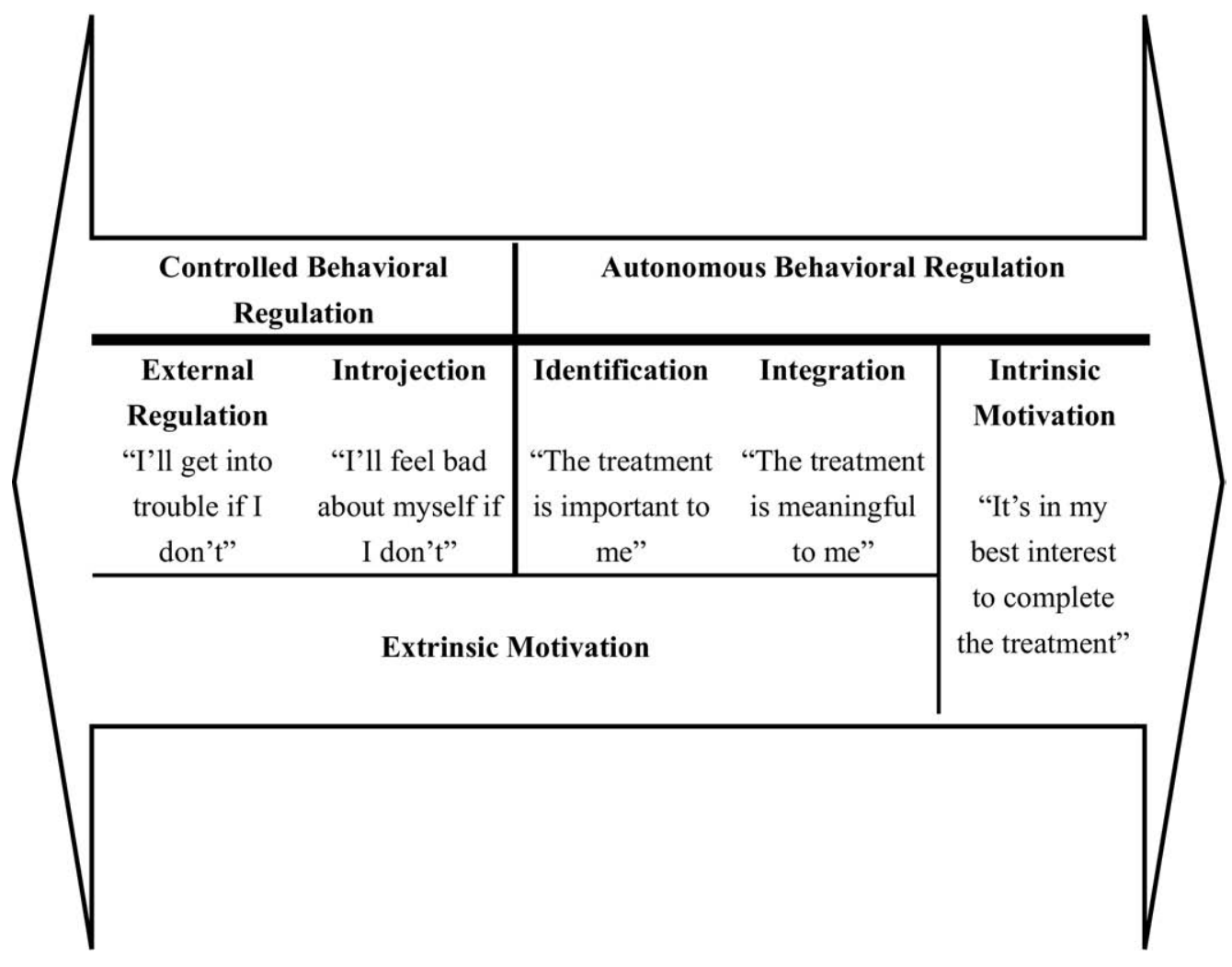

Low self-determination

High self-determination

Fig 1. The self-determination continuum.

regulation) and 2 of which are related to autonomous motivation (identified and integrated regulation).

External regulation is the most controlled form of extrinsic motivation and exists when the behavioral goals are to satisfy an external contingency (eg, avoid punishment or obtain rewards). Introjected regulation is still a controlling form of regulation but is somewhat more autonomous than external regulation. Whereas external regulation refers to motivation brought about by external pressures, introjected regulation involves pressure from within the individual; for example, behaviors performed in order to avoid feelings of guilt or to enhance self-worth.

Identified regulation is more autonomous than introjected regulation and refers to a person performing an action because he or she values the benefits that will be derived. Integrated regulation is the most autonomous form of extrinsic motivation and exists when one's goals are in line with one's core values and sense of self.

To summarize, controlled motivation refers to behaviors that are the result of external or internal pressure. In contrast, autonomous motivational types include intrinsic motivation and extrinsic motivation that is underpinned by a desire to obtain benefits that are highly valued. In the health care context, patients who have adopted controlled motivation will participate in treatment because of an external force (eg, "I'll get into trouble if I don't") or an internal conflict (eg, "I'll feel bad about myself if I don't"). ${ }^{11}$ In contrast, patients with autonomous motivation will identify the goals of treatment (eg, "it's in my best interests to complete treatment") and feel competent concerning the outcomes of the rehabilitation pro- cess (eg, "I feel like it's the best way to help myself"). ${ }^{11}$ These 2 forms of treatment motivation, controlled versus autonomous, have been differentially related to the adherence of patients who undertake treatments in a variety of medical contexts.

Autonomous motivation towards treatment has been positively associated with adherence to medical regimens among people with chronic illnesses, ${ }^{8,9}$ attendance/involvement in an addiction treatment program, ${ }^{6,10,11}$ and long-term maintenance of weight loss among morbidly obese patients. ${ }^{7}$ In contrast, controlled treatment motivation has either minimal or negative effects on adherence. ${ }^{7,8,10,22}$

Apart from motivation, social influence is another factor that has been suggested to influence adherence to treatment. Previous studies found that the degree to which a patient is satisfied with the support and help received from others was associated with physiotherapists' ratings of adherence, treatment protocol completion, and attendance at rehabilitation sessions. ${ }^{16,18,20}$ SDT also suggests that social contextual factors will influence motivation and resultant behavior. According to SDT, an autonomy-supportive environment, where individuals perceive that they have been provided with opportunities for choice and options, respect and acknowledgment of their opinions and feelings, and meaningful rationale behind recommendations, will result in greater autonomous motivation and less controlled motivation. ${ }^{13,21,23}$

Health care practitioners' autonomy-supportive behaviors have been positively correlated with adherence to prescribed medical regimens. ${ }^{8,9}$ Furthermore, evidence from intervention studies also indicates that smokers who receive autonomy- 
supportive persuasions from physicians to quit smoking (eg, "smoking is a matter of choice, but there are important healthrelated reasons for refraining") have increased abstinence from smoking than those who receive a controlling style of persuasion (eg, "you should refrain from smoking to prevent horrible diseases"). ${ }^{6,10,12}$ Also, autonomous motivation serves as the mediator for the relationship between autonomous support and treatment compliance. ${ }^{8-10}$ However, there is limited evidence regarding the psychosocial factors that may influence other types of patients' motivation and adherence to recommended treatment. For example, little is known about orthopedic surgery patients' motivation and adherence to rehabilitation programs prescribed by their health professionals.

Based on the tenets of SDT, we hypothesized that patients' perceptions of their physiotherapists' autonomy-supportive behaviors and patients' autonomous motivation to complete their rehabilitation would be positively associated with a greater adherence to home-based rehabilitation exercise. Controlled motivation was expected to show a negative relationship with adherence. In line with SDT tenets,,${ }^{9,13,23}$ we further hypothesized that patients' motivation would mediate the relationship between their physiotherapists' autonomy-supportive behaviors and patients' adherence.

\section{METHODS}

\section{Participants and Procedures}

All 246 patients who underwent ACL reconstructions in a northern district Hong Kong hospital between 2005 to 2008 were invited to participate in the study. After receiving full details of the study, 115 patients $(46.75 \%$ response rate) agreed to participate. The participants were 94 males (mean age \pm SD, $27.05 \pm 3.99 \mathrm{y}$ ) and 21 females (mean age $\pm \mathrm{SD}, 23.38 \pm 4.01 \mathrm{y}$ ) who underwent ACL reconstruction at least 6 months before the study (range, .50-3.0y; mean interval $\pm \mathrm{SD}, 1.77 \pm .80 \mathrm{y}$ ). The study was approved by a university research ethics board, and participants gave informed consent by indicating they fully understood the voluntary nature of their participation, their right to withdraw from the study, and the confidentiality of the data. Participants completed questionnaires regarding their rehabilitation experience without the presence of any medical staff. Before their ACL rupture, all participants had been involved in sports, including soccer $(54.4 \%)$, basketball $(28.1 \%)$, and others $(17.5 \%)$, for a mean \pm SD of $8.48 \pm 6.91$ years. Participants ruptured their ACL during training or competition in their sport and had no history of knee injury before this incident.

\section{Measures}

The HCCQ, ${ }^{7}$ a 15 -item instrument, measured the degree to which patients perceived their physiotherapist was autonomy supportive. The 13-item TSRQ ${ }^{\dagger}$ measured participants' autonomous and controlled motives to continue their rehabilitation program in the first 6 months after their surgery. The HCCQ and TSRQ were initially developed to measure the perceived autonomy support and treatment motivation of obese patients, ${ }^{7}$ and were shown to be reliable and valid across a variety of health care contexts. ${ }^{6,8-10,24}$ In both questionnaires, participants responded using 7-point Likert scales ranging from "strongly disagree" (1) to "strongly agree" (7).

Finally, to assess patients' self-reported rehabilitation adherence we considered items from the Sport Injury Rehabilitation Adherence Scale. ${ }^{25}$ This questionnaire was designed to measure physiotherapists' perceptions of their patients' rehabilitation adherence. As a result, not all of the items were appropri- ate for a scale designed to measure patient perceptions, as was needed in the current study. We also considered items from the Patient Self-Report Scales of Their Home-Based Rehabilitation Adherence. ${ }^{26}$ This scale is a patient self-report measure, but some of the items referred to rehabilitation protocols that were not relevant to ACL reconstruction patients throughout the 6-month postsurgery interval (eg, the application of ice). We concluded that 3 concepts from the 2 questionnaires were relevant to ACL reconstruction patients: (1) rehabilitation completion, (2) rehabilitation effort, and (3) avoidance of specific activities. We then adapted items from the 2 questionnaires and included them in the survey. These items were "carry out rehabilitation exercise recommended by your physiotherapists," using a Likert scale ranging from "complete none" (1) to "complete all" (7); "make an effort to do the rehabilitation exercises recommended by your physiotherapist," with a Likert scale ranging from "minimum effort" (1) to "maximum effort" (7); and "refrain from undertaking the sporting and daily activities that your physiotherapists advised not to do," with "avoid none" (1) and "avoid all" (7) as anchors. Patients were asked to recall their behaviors in the first 6 months after their surgery. Possibly because of the negative wording of the item, low interitem correlation $(r=.02-.09)$ was found in relation to the scores from the third item. As a result, we removed this item from subsequent analyses.

Participants were asked to respond according to their experiences during the first 6 months after their surgery. Clear instructions (ie, "in the first 6 months after the surgery,") were included to facilitate accurate recalls. Also, participants were advised to close their eyes and recall their rehabilitation experiences before they completed the questionnaire.

\section{Chinese Translation}

The English items were translated into Chinese (the participants' native language) by a pair of bilingual individuals. These items were then back-translated into English by a separate bilingual pair. Both pairs then met with a third pair to resolve any discrepancies and finalize the translation. The translated questionnaire was then pilot tested and deemed comprehensible by $10 \mathrm{ACL}$ reconstruction patients.

\section{Analysis}

After replacing the missing data $(<3 \%$ missing $)$ using an expectation maximization algorithm, we used confirmatory factor analysis to test the measurement models for the HCCQ and TSRQ, and examined the descriptive statistics associated with each variable. We then used SEM to test the hypothesis that treatment motivation would mediate the relationship between autonomy support and rehabilitation adherence. Because of the limited sample size in this study, we used item parceling to reduce the number of parameters in the SEM models. Item scores for autonomy support, controlled treatment motivation, and autonomous treatment motivation were parceled into 5, 4, and 3 indicators, respectively. These indicators were entered into the model to estimate the associated latent factors. The adherence latent factor was formed using the observed scores from the 2 items described in the Methods section. To examine the fit of the data to the hypothesized model, we followed convention and examined a range of indexes. ${ }^{27} \mathrm{We}$ used RMSEA and SRMR as 2 absolute fit indices, and TLI and CFI as 2 incremental fit indices. TLI and CFI scores of .90 or higher, as well as SRMR and RMSEA scores of .08 or less, are typically considered adequate, while RMSEA scores between .08 and .10 represent marginal fit. $\mathrm{Hu}$ and Bentler $^{27}$ have proposed more stringent cutoff criteria (eg, TLI and CFI, $\geq .95$; 


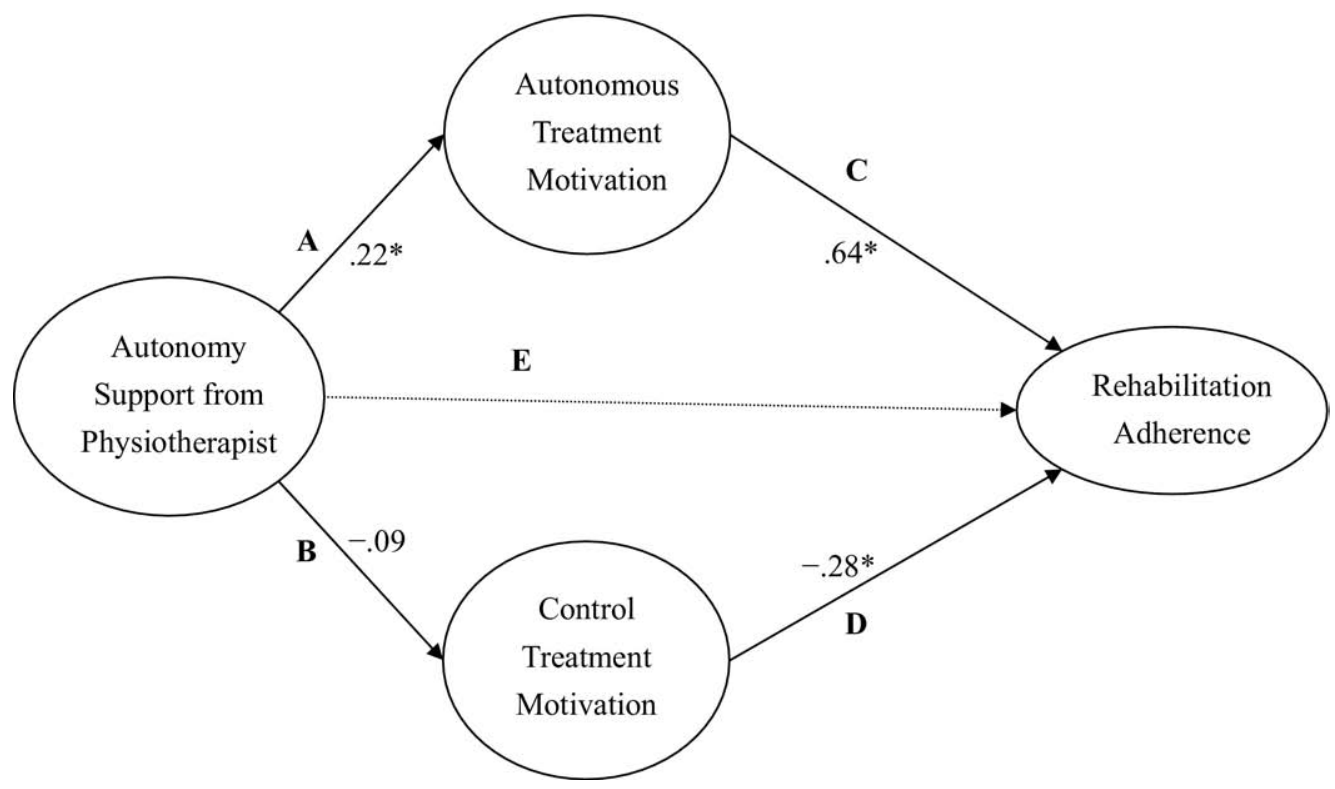

Fig 2. Self-determination model for rehabilitation adherence after ACL reconstruction. Only path estimates from the mediation model are shown; ${ }^{*} P<.05$. The additional vector $(E)$ with a dotted line was only present in direct effect and combined effects models.

RMSEA, $\leq .06 ;$ SRMR, $\leq .08)$. We considered these criteria as evidence of very good fit.

Mediation analysis ${ }^{28}$ was used by first testing a direct effect model, then a mediation model, and last a combined effects model (fig 2). In the direct effect model, autonomy support was the only variable specified to predict adherence. In the mediation model, autonomous and controlled treatment motivation served as mediators of the relationship between autonomy support and adherence. In the combined effects model, autonomy support was added as a direct predictor of adherence on top of the mediation model. Mediation would be shown by (1) the presence of a significant autonomy support $\rightarrow$ adherence path (path E in fig 2) in the direct effect model, (2) acceptable fit in the mediation model, (3) no significant improvement in fit in the combined effects model relative to the mediation model $(P<.05)$, and (4) a significant reduction in the strength of the autonomy support $\rightarrow$ adherence path (path $E$ in fig 2 ) in the combined effects model. If complete mediation were present, this latter path would be nonsignificant in this final model $(P>.05)$

\section{RESULTS}

\section{Preliminary Analyses}

After reverse coding item 13 of the HCCQ, all the item scores on each scale were summed before examining the descriptive statistics. Mean \pm SD scores for physiotherapists' autonomy support (5.39 \pm 1.12 ; range, $1.40-7.00)$, autonomous treatment motivation $(5.36 \pm 1.04$; range, $1.80-7.00)$, and adherence (5.50 1.16 ; range, 2.50-7.00) were above the scale midpoint. Controlled motivation mean \pm SD scores were somewhat lower $(3.52 \pm 1.22$; range, $1.00-6.50)$. All variables in the study showed an acceptable level of variance, thereby ensuring that relationships between variables in subsequent analyses would not be artificially deflated. Alpha values for the HCCQ and TSRQ scales ranged from .73 to .95. The correlation between the 2 adherence items was 0.8. These results suggested that the questionnaires produced internally consistent scores.

There were no multivariate outliers $(P<.001)$ and no evidence of univariate or multivariate nonnormality (Mardia's normalized kurtosis coefficient, 4.83). As a result, we used maximum likelihood estimation for all SEM analyses. Despite the small sample size, most fit indexes from the preliminary confirmatory factor analyses supported the factorial validity of the HCCQ and TSRQ (see table 1 for details). There was a degree of misfit (eg, RMSEA>.08) for both scales; however, there was not enough evidence to warrant respecification of these measurement models. Further investigation, with larger samples, is needed to provide more conclusive evidence concerning the validity of these measures in orthopedic surgery patients. After considering the largely supportive factorial validity evidence and the strong reliability coefficients, we deemed the psychometric properties of the 3 questionnaires in this study sufficient to continue with the main analysis.

\section{Main Analysis}

Overall, both the mediation and combined effects models showed generally acceptable fit. In both models, the RMSEA indicated that the fit was only marginally acceptable; however, evidence from the other 3 fit indexes (CFI, TLI, SRMR) suggested that the model fit adequately. Complete details regarding the SEM analyses can be seen in table 1 . In the direct effect model, autonomy support was found to predict adherence $(\gamma=.26, P<.05)$, and thus the first criterion for mediation was fulfilled. The mediation model showed generally acceptable fit to the data and accounted for substantial variance in adherence $\left(R^{2}=.28\right)$. Rehabilitation adherence was predicted positively by autonomous treatment motivation $(\beta=.62, P<.05)$, and negatively predicted by controlled treatment motivation $(\beta=-.26, P<.05)$. Autonomous treatment motivation was associated positively with autonomy support $(\gamma=.22, P<.05)$, but the relationship between autonomy support and controlled treatment motivation was not significant $(\gamma=-.09, P<.05)$. 
Table 1: Fit Statistics and Standardized Path Coefficients for Structural Equation Models

\begin{tabular}{|c|c|c|c|c|c|c|c|c|c|c|c|c|}
\hline \multirow[b]{2}{*}{ Model } & \multirow[b]{2}{*}{$\chi^{2}$} & \multirow[b]{2}{*}{$d f$} & \multirow[b]{2}{*}{ TLI } & \multirow[b]{2}{*}{ CFI } & \multirow[b]{2}{*}{ SRMR } & \multirow[b]{2}{*}{$\begin{array}{l}\text { RMSEA } \\
(90 \% \mathrm{Cl})\end{array}$} & \multicolumn{2}{|c|}{$\begin{array}{l}\text { Aut Support } \rightarrow \mathrm{Tx} \\
\text { Motivation }\end{array}$} & \multicolumn{2}{|c|}{ Tx Motivation $\rightarrow$ Adh } & \multirow[b]{2}{*}{$\frac{\text { Aut Support } \rightarrow \text { Adh }}{\text { (e) }}$} & \multirow[b]{2}{*}{ Adh $R^{2}$} \\
\hline & & & & & & & $\begin{array}{l}\text { Aut Motv } \\
\text { (a) }\end{array}$ & $\begin{array}{l}\text { Cont Motv } \\
\text { (b) }\end{array}$ & $\begin{array}{l}\text { Aut Motv } \\
\text { (c) }\end{array}$ & $\begin{array}{l}\text { Cont Motv } \\
\text { (d) }\end{array}$ & & \\
\hline Aut support (CFA) & 259.54 & 90 & .90 & .91 & .04 & $.13(.11-.14)$ & NA & NA & NA & NA & NA & NA \\
\hline Tx Motv (CFA) & 47.54 & 26 & .87 & .96 & .05 & $.09(.04-.12)$ & NA & NA & NA & NA & NA & NA \\
\hline Direct effect & 181.86 & 75 & .89 & .91 & .13 & $.11(.09-.13)$ & NA & NA & NA & NA & $.26^{*}$ & .07 \\
\hline Mediation & 154.75 & 72 & .91 & .93 & .08 & $.10(.08-.12)$ & $.22 *$ & -.09 & $.64 *$ & $-.28^{*}$ & NA & .28 \\
\hline Combined effects & 154.66 & 71 & .91 & .93 & .08 & $.10(.08-.12)$ & $.21 *$ & -.09 & $.63 *$ & $-.27 *$ & -.03 & .28 \\
\hline
\end{tabular}

Abbreviations: Adh, adherence; Aut, autonomy; CFA, confirmatory factor analysis; Cl, confidence interval; Cont, controlled; Motv, motivation; NA, not applicable; Tx, treatment.

*Indicates that the standardized path coefficient was significantly different from zero $(P<.05)$.

Analysis of the combined effects model showed a significant indirect effect of autonomy support on adherence $(\gamma=.16$, $P<.05)$, which accounted for $82.20 \%$ of the total effect $(\gamma=.19, P<.05)$. Also, the chi-square difference test showed that the combined effects model did not fit the data better than the mediation model $(P>.05)$. Finally, the significant path from autonomy support to adherence in the direct effect model was reduced to a nonsignificant value in the combined effects model. Taken together, these results indicated that autonomous treatment motivation fully mediated the effect of physiotherapists' autonomy-supportive behaviors on patients' adherence.

\section{DISCUSSION}

In this study, we investigated the relationships between physiotherapists' autonomy-supportive behaviors, patients' treatment motivation, and rehabilitation adherence. Consistent with our hypothesis, autonomy support from physiotherapists had a positive relationship with rehabilitation adherence, and this effect was mediated by patients' treatment motivation. Autonomous motivation was positively related to adherence, while controlled motivation was negatively correlated with adherence.

Deci and Ryan ${ }^{13,21}$ suggested that long-term maintenance of behaviors depends on one's acceptance of the values of the behavior, and behavior underpinned by controlled regulations will not be maintained. Consistent with previous studies in health care context, ${ }^{7-9,11}$ our results suggested that the sources of motivation may influence a patient's compliance with treatment recommendations. ACL reconstruction patients who adopted higher autonomous motivation, as opposed to controlled motivation, were more likely to report that they had completed their home-based rehabilitation exercises. Treatment motivations for undertaking rehabilitation protocol after ACL reconstruction in this study accounted for $28 \%$ of variance in compliance to treatment. These results are in line with studies of other health-related behaviors, such as weight maintenance, ${ }^{7}$ smoking cessation, ${ }^{6,10}$ and diabetes glucose control, ${ }^{9}$ where motivation accounted for $25 \%$ to $43 \%$ of variance.

As noted, autonomous motivation appeared adaptive, and controlled treatment motivation maladaptive, in relation to adherence to postsurgery rehabilitation treatment. The next logical inquiry is therefore how health professionals can promote this adaptive, autonomous treatment motivation and prevent the development of treatment-compromising controlled motivation. In this study, physiotherapists' autonomy-supportive behaviors were associated with autonomous treatment motivation, and autonomous treatment motivation was the mediator for the positive association between autonomy support and patients' treatment adherence. These findings were consistent with previous SDT-based studies in health care settings, ${ }^{6-9}$ and other non-SDT-based investigations, suggesting that social support from medical care providers is an important factor that influences treatment adherence. ${ }^{16,18,20,29}$

Our study provides initial evidence that ACL reconstruction patients who believed their physiotherapist facilitated their understanding of treatment, provided treatment options, and explained the rationale behind treatment were more likely to report autonomous motivation and greater adherence. However, we did not find the hypothesized significant negative relationship between autonomy-supportive behavior and controlled treatment motivation. It may be that the presence of controlling practitioner behaviors, rather than the mere absence of autonomy-supportive behaviors, is responsible for the development of controlled treatment motivation. The measure of physiotherapists' behavior that we used (HCCQ) ${ }^{7}$ did not include items to measure these types of behaviors, and thus future research is needed to determine the role of controlling practices (eg, coercive advice) on patient motivation.

\section{Limitations and Future Research Directions}

There are clear methodological limitations associated with this study. First, patients' retrospective, self-reported recall of their experiences in the 6 months after their surgery was subject to memory loss and various forms of bias (eg, social desirability). Variation in participants' recall precision may have introduced error variance and reduced the strength of associations between the variables in this study. However, follow-up regression analyses ${ }^{30}$ indicated that the postsurgery interval did not moderate any of the relationships in the mediation model (ie, cross-product interaction terms were not significantly different from zero, $P>.20$; contact the authors for full details). As a result, this limitation did not appear to be a substantial concern in this study. Second, our sample contained only athletes who had ruptured their ACL during sport training or competition. Future studies could include patients recovering from injuries sustained during a wider variety of activities, including noncompetitive recreational pursuits and occupational activities. Moreover, the response rate in this study was approximately $50 \%$. Although the rate was comparable to that reported in previous studies of patients' adherence to long-term medical regimens, ${ }^{8,9}$ it is still possible that patients who were willing to participate in this study had more positive rehabilitation experiences than those who chose not to participate. This sampling bias is unlikely to have inflated the size of the relationships we observed; rather the potential restriction of variation in scores would have reduced the strength of these associations. ${ }^{9}$ However, we must be cautious concerning the external validity of our results and underscore the importance 
of future research employing a prospective design that could help ensure a higher response rate.

A prospective design involving an autonomy support intervention would also help to overcome the third limitation to our study; namely, the retrospective design, which limited our ability to infer that autonomy support caused patients to adopt autonomous motives and adhere to their rehabilitation programs. Randomized controlled trials involving training to enhance health care professionals' autonomy-supportive behaviors have produced large increases in patient adherence and improvements in blindly assessed treatment outcomes in other areas of health care. ${ }^{6,10,12}$ These types of studies within the context of ACL reconstruction surgery would provide stronger evidence concerning the impact of autonomy-supportive behaviors on patients' treatment motivation and adherence. Importantly, this research should not only investigate the efficacy of interventions designed to enhance physiotherapists' autonomy support, but should also examine the impact of training other medical practitioners (eg, surgeons) and patients' significant others (eg, spouse or parents) to act in an autonomysupportive manner. $16,30,31$

\section{CONCLUSIONS}

The present study provides initial evidence that $\mathrm{SDT}^{13,21}$ can help to explain patients' rehabilitation adherence after ACL reconstruction surgery. Our results suggest that autonomy support from physiotherapists and autonomous treatment motivation are positive predictors, while controlled treatment motivation is a negative predictor of patients' adherence to their postsurgery rehabilitation. These findings provide an empirical basis to support future investigations of autonomy support training designed to increase patients' postsurgery adherence to prescribed rehabilitation exercises.

\section{References}

1. Schneider S, Seither B, Tonges S, Schmitt H. Sports injuries: population based representative data on incidence, diagnosis, sequelae, and high risk groups. Br J Sports Med 2006;40:334-9.

2. Shelbourne KD, Nitz P. Accelerated rehabilitation after anterior cruciate ligament reconstruction. Am J Sports Med 1990;18: 292-9.

3. Rolf CG, Chan KM. Knee injuries. In: Chan KM, Micheli L, Smith A, et al editors. International Federation of Sports Medicine: team physician manual. 2nd ed. Hong Kong: International Federation of Sports Medicine; 2006. p 373-406.

4. Ice R. Long term compliance. Phys Ther 1985;65:1832-9.

5. Sluijs EM, Kok GJ, van der Zee J. Correlates of exercise compliance in physical therapy. Phys Ther 1993;73:771-82.

6. Williams GC, Gagne M, Ryan RM, Deci EL. Facilitating autonomous motivation for smoking cessation. Health Psychol 2002; 21:40-50.

7. Williams GC, Grow VM, Freedman ZR, Ryan RM, Deci EL. Motivational predictors of weight loss and weight-loss maintenance. J Pers Soc Psychol 1996;70:115-26.

8. Williams GC, Freedman ZR, Deci EL. Supporting autonomy to motivate patients with diabetes for glucose control. Diabetes Care 1998;21:1644-51.

9. Williams GC, Rodin GC, Ryan RM, Grolnick WS, Deci EL. Autonomous regulation and long-term medication adherence in adult outpatients. Health Psychol 1998;17:269-76.

10. Williams GC, Cox EM, Kouides R, Deci EL. Presenting the facts about smoking to adolescents-effects of an autonomysupportive style. Arch Pediatr Adolesc Med 1999;153:959-64.
11. Ryan RM, Plant RW, O'Malley S. Initial motivations for alcohol treatment: relations with patient characteristics, treatment involvement, and dropout. Addict Behav 1995;20:279-97.

12. Williams GC, McGregor HA, Sharp D, et al. Testing a selfdetermination theory intervention for motivating tobacco cessation: supporting autonomy and competence in a clinical trial. Health Psychol 2006;25:91-101.

13. Deci EL, Ryan RM. Intrinsic motivation and self-determination in human behavior. New York: Plenum; 1985.

14. Fisher AC. Counseling for improved rehabilitation adherence. In: Ray R, Wiese-Bjornstal DM, editors. Counseling in sports medicine. Champaign: Human Kinetics; 2001. p 275-309.

15. Jensen GM, Lorish CD. Promoting patient cooperation with exercise programs: linking research, theory, and practice. Arthritis Care Res 1994;7:181-9.

16. Duda JL, Smart AE, Tappe MK. Predictors of adherence in the rehabilitation of athletic injuries: an application of personal investment theory. J Sport Exerc Psychol 1989;11:367-87.

17. Brewer BW, Cornelius AE, Van Raalte JL, et al. Age-related differences in predictors of adherence to rehabilitation after anterior cruciate ligament reconstruction. J Athl Train 2003; 38:158-62.

18. Brewer BW, Van Raalte JL, Cornelius AE, et al. Psychological factors, rehabilitation adherence, and rehabilitation outcome after anterior cruciate ligament reconstruction. Rehabil Psychol 2000; 45:20-37.

19. Fields J, Murphey M, Horodyski MB, Stopka C. Factors associated with adherence to sport injury rehabilitation in college-age recreational athletes. J Sport Rehab 1995;4:172-80.

20. Levy AR, Polman RC, Clough PJ. Adherence to sport injury rehabilitation programs: an integrated psycho-social approach. Scand J Med Sci Sports 2008;18:798-809.

21. Deci EL, Ryan RM. The "what" and "why" of goal pursuits: human needs and the self-determination of behavior. Psychol Inq 2000;11:227-68.

22. Williams GC, Minicucci DS, Kouides RW, et al. Self-determination, smoking, diet and health. Health Edu Res 2002;17:512-21.

23. Ryan RM, Deci EL. Self-determination theory and the facilitation of intrinsic motivation, social development, and well-being. Am Psychol 2000;55:68-78.

24. Williams GC, Deci EL. Activating patients for smoking cessation through physician autonomy support. Med Care 2001;39:813-23.

25. Kolt GS, Brewer BW, Pizzari T, Schoo AM, Garrett N. The Sport Injury Rehabilitation Adherence Scale: a reliable scale for use in clinical physiotherapy. Physiotherapy 2007;93:17-22.

26. Bassett SF. The assessment of patient adherence to physiotherapy rehabilitation. N Z J Physiother 2003;31:60-6.

27. $\mathrm{Hu} \mathrm{L}$, Bentler PM. Cutoff criteria for fit indexes in covariance structural analysis: conventional criteria versus new alternatives. Structural Equation Modeling 1999;6:1-55.

28. Baron RM, Kenny DA. The moderator mediator variable distinction in social psychological-research-conceptual, strategic, and statistical considerations. J Pers Soc Psychol 1986;51:1173-82.

29. Cameron C. Patient compliance: recognition of factors involved and suggestions for promoting compliance with therapeutic regimens. J Adv Nurs 1996;24:244-50.

30. Aiken LS, West SG. Multiple regression: testing and interpreting interactions. Newbury Park: Sage; 1991.

31. Pulliam C, Gatchel RJ, Robinson RC. Challenges to early prevention and intervention: personal experiences with adherence. Clin J Pain 2003;19:114-20. 